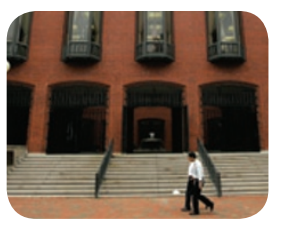

\author{
Inequitable \\ conduct attacks \\ may subside $p 672$
}

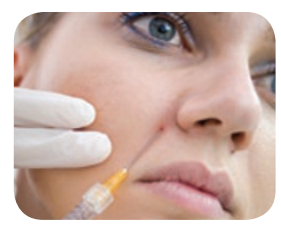

FDA approves first

cell cosmetic $p 674$

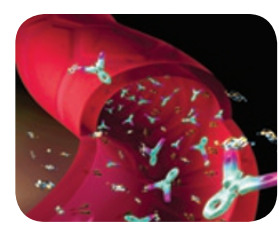

Avastin rejected by

FDA panel-again

p676

\title{
E. coli crisis opens door for Alexion drug trial
}

This spring's outbreak of a virulent form of Escherichia coli in Germany and France provoked a rapid response from public health authorities and the research community. Not only did the response represent a triumph of global collaboration in rapidly characterizing the Shiga toxin-producing strain but it also prompted an on-the-fly clinical trial of one of the world's most expensive biotech drugs-Alexion's humanized monoclonal antibody Soliris (eculizumab) - previously approved for the rare disease paroxysmal nocturnal hemoglobinuria (PNH). As thousands fell ill with the enterohemorrhagic E. coli (EHEC) strain O104:H4 from eating tainted food-a substantial fraction developing potentially fatal hemolytic uremic syndrome (HUS) - German and then French doctors turned to Soliris, which prevents the cleavage of complement component 5 (C5) and activation of the hemolytic cascade.

The potential of Soliris to treat those affected by HUS was highlighted by a publication rushed to print on May 25 by the New England Journal of Medicine (364, 2561-2563, 2011). In that study, the drug was prescribed off-label with positive results to treat HUS in three unrelated cases of EHEC. The outbreak thus presented Alexion, which has a market cap of nearly $\$ 10$ billion, with an opportunity to conduct a large trial with rapid patient recruitment. Germany only has around 50 HUS patients in a typical year, says nephrologist Franz Schäfer of the University of Heidelberg and an author on the NEJM paper: "It's a huge occasion for the company. It saves them a lot of time and a lot of money."

In Germany and France, the E. coli strain sickened over 6,000 people and caused HUS in at least 300 cases, with at least 36 fatalities. As requests for Soliris mounted, the Cheshire, Connecticut-based biotech elected to provide the drug free on a compassionate-use basis, at the same time approaching regulatory authorities for guidance.

Soliris is an expensive drug-treating $\mathrm{PNH}$ costs from $\$ 400,000$ to over $\$ 500,000$ a year over the entire course of the patient's life. There are only 8,000-10,000 PNH patients in North America and Western Europe, only a fraction of which are sufficiently hemolytic to qualify for therapy. Alexion's decision not to charge for the drug in HUS could pay dividends by opening up a new indication. "They [Alexion] thought that this is a really large-scale use of the compound in a new potential indication and they should make a trial out of it," says Schäfer.

Soliris suppresses abnormal activation of C5, which damages red blood cells. PNH patients have a mutation in PIGA, a gene that encodes glycosylphosphatidylinositol, which helps anchor proteins to the membrane. When the mutation is present, cell membranes are more prone to complementinduced damage. As a result, red blood cells break down too early and leak hemoglobin into urine. If complement damages too many red blood cells, the kidneys can fail. In EHEC patients, Shiga toxin produced by E. coli O104:H4, instead of the PIGA mutation, causes abnormal complement activation. Though the origin of the complement activation differs in EHEC and PNH, the complement overactivity is similar, so suppressing it with Soliris may help HUS patients in the same way it helps PNH patients. In April, Alexion filed for approval of the drug for atypical HUS in the US and EU, and two months later the US Food and Drug Administration granted priority review in the same indication.

By June 20, in collaboration with Alexion, Germany's biologics drug regulatory agency, the Paul Ehrlich Institute in Langen, launched a clinical trial to compare uses of Soliris in HUS. Unfortunately, because doctors began administering Soliris to all their sickest patients before the trial began, there is no good way of comparing the drug's effect to other treatments or to no treatment at all.

Regulatory authorities have also established a national German registry through the Paul Ehrlich Institute and later through the European Center for Disease Prevention and Control in Stockholm. "The whole system was preconditioned by the swine flu epidemic...especially the European Center for Disease Control," says nephrologist Jan T. Kielstein at the Hannover Medical School, in Hannover, Germany. Researchers at Saarland University in Saarbruecken, Germany, for example, shared a genetic database for EHEC-HUS, to speed up other researchers' work on the bacterium.

Kielstein says that unity among researchers, regulators and the biotech drugmaker during the crisis was "spectacular." When Alexion requested the trial, the Paul Ehrlich Institute devoted all its staff and worked through two weekends to obtain approval for the trial by June 20-about a quar-

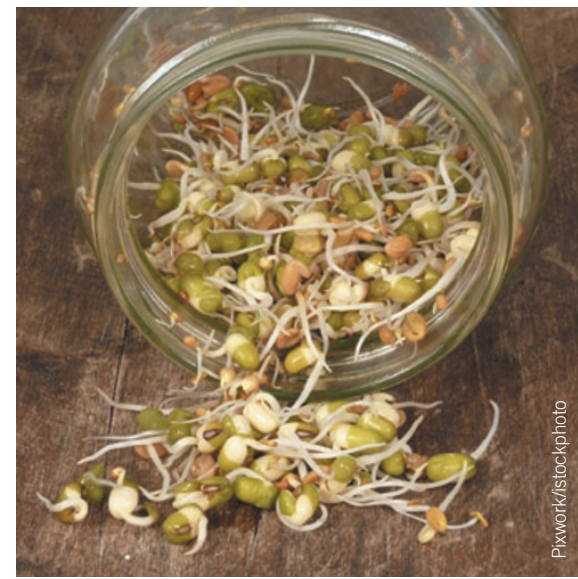

Seeds used for sprouting were the likely source of the E. coli outbreak in Germany and France. Alexion stepped in to provide an expensive antibody treatment to those who fell ill.

ter the typical time, according to the institute's president Klaus Cichutek. The trial will follow two groups of patients through treatment lasting 8 weeks and 26 weeks, respectively, as it is too late to include a control arm.

Analyst Stephen Willey at Stifel Nicolaus, New York, says: "I don't view the clinical trial as a potentially large windfall for the company...given the episodic nature of incidence and the likely short treatment duration implied by anecdotal data to date." He agrees, however, that "Alexion could not have asked for a better pre-launch campaign to increase awareness." Last month, Soliris announced Q2 sales that grew $\sim 12 \%$ over the prior quarter to $\$ 185.7$ million. Alexion improvised its response to the E. coli outbreak, according to the company's spokesperson Irving Adler. Although the firm was aware of the NEJM study, its 2010 report to stockholders contains no mention of plans to treat HUS. Little more than six months later, Alexion was supplying French doctors with the drug "on the same basis" as those in Germany, Adler says. The company holds an 18-month supply on hand from two production facilities and should be able to keep up with demand related to this E. coli outbreak. Adler adds that it is too early for Alexion to comment on the commercial implications of the E. coli HUS trial.

Lucas Laursen Zurich 